\title{
Classifying Spaces for Quantum Principal Bundles
}

\author{
Andreas Müller * \\ I.H.E.S., 35 route de Chartres, F-91440 Bures-sur-Yvette, France
}

Received December 13, 1991; in revised form March 23, 1992

\begin{abstract}
A construction of a quantum analogue of principal bundles is discussed. Deformations of quantum groups in the sense of Woronowicz allow to relax the condition of local triviality of a principal bundle; the fibres need not be all identical any longer. This leads to deformations of structure group and bundles. There is still a classifying space in the sense that homotopy classes of bundles are classified by homotopy classes of maps from the base space into the classifying space.
\end{abstract}

\section{Table of Contents}

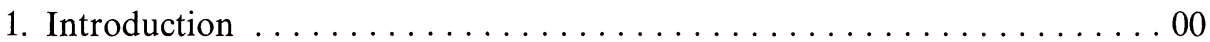

2. Coequivariant Maps ........................ 00

3. Deformations of Quantum Groups and Their Character Groups . . . . . 00

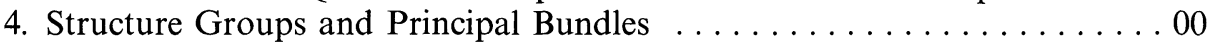

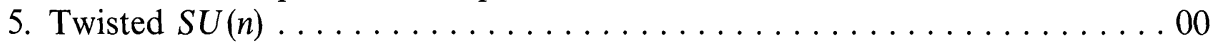

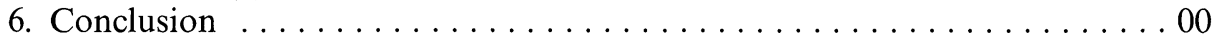

\section{Introduction}

In particle physics as well as in differential geometry, principal fibre bundles play the key role of making precise the notion of local symmetry. One may ask whether the concept can be generalized to include quantum groups as fibres.

The proper setting for this question would of course be noncommutative geometry, but it seems quite difficult to formulate a notion analogous to local triviality in the noncommutative setting. If one formulates the theory in completely categorical terms in the category of topological spaces, one needs categorical products. One cannot translate these directly to the category of $C^{*}$-algebras, they

\footnotetext{
* email: afm@frihes61.bitnet
} 
would have to be replaced by coproducts in the category of $C^{*}$-algebras, but the tensorproduct, which is the coproduct in the subcategory of commutative $C^{*}$ algebras, is not the coproduct in the whole category.

G. Maltsiniotis has come upon a similar problem in his recent definition of quantum groupoids [4]. A groupoid is a category in which all morphisms are isomorphisms, a topological groupoid is a groupoid such that the set of objects and the set of morphisms are topological spaces and the structure maps (source and range of a morphism, composition of morphisms, identity morphism) are continuous. A principal fibre bundle is a groupoid where source and range maps coincide and all endomorphism groups are isomorphic, the base space is the space of objects. In Maltsiniotis' definition, the algebra corresponding to the space of objects is a commutative algebra, i.e. a "real" space. We therefore restrict ourselves to topological spaces as base spaces.

We should probably be less strict about local triviality for the following reason. In the topological theory, we would like the fibre to vary continuously with its base point. For Lie groups as fibres there is not much space to move, Lie groups are completely rigid, hence all the fibres are the same; only the way they are put together matters. By enlarging our set of fibres a bit, e.g. in allowing quantum deformations of Lie groups, we may get different fibres over different points.

In Chap. 2 we discuss the most obvious generalization of the notion of a $G$-map $G \rightarrow G$, where $G$ is considered as a right $G$-space. These are the endomorphisms of the "algebra of functions" on the quantum group considered as a right comodule over itself. For groups we know that all such maps are invertible and are given by left multiplication by some group element. A similar statement holds for Hopf algebras. This means that the right comodule maps form a group which we can identify with the classical points of the quantum group.

Chapter 3 is devoted to deformations of quantum groups and representations. In Chap. 4 we construct bundles with quantum group fibres and their classifying spaces. For this we mimic the classical construction, taking right comodule homomorphisms for the transition functions. This restricts our construction to a semiclassical situation, but it is the only way that allows to retain the comodule structure. Chapter 5 presents as an example the quantum group $S U_{\mu}(n)$ of $[8]$.

\section{Coequivariant Maps}

Let $G$ be a topological group, which we also consider as a right $G$-space, $G$ acting by right multiplication. Then every continuous $G$-equivariant map $f: G \rightarrow G$ is given by $f(g)=h g$, where $h \in G$ is some group element. Similar statements hold for the left action of $G$ upon itself. In this section we generalize these observations to quantum groups.

2.1. Hopf Algebras and Comodules. Let $H$ be a Hopf algebra over some fixed field $k$, with multiplication $m: H \otimes H \rightarrow H$, unit $\eta: k \rightarrow H$, comultiplication $\Delta: H \rightarrow$ $H \otimes H$, counit $\varepsilon: H \rightarrow k$ and antipode $i: H \rightarrow H$ (we use the notations of [5]). All tensor products are over $k$ unless explicitly indicated. Also all homomorphisms of algebras are (unital) homomorphisms over $k$. 
A right- $H$-comodule is a $k$-vectorspace $E$ with a linear map $\delta_{E}: E \rightarrow E \otimes H$, the coaction, such that the diagrams
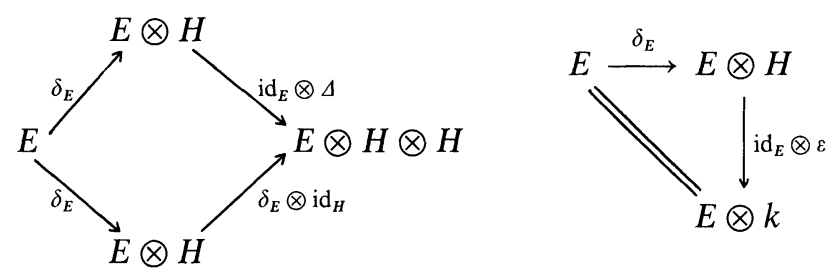

commute. A linear map $f$ between comodules $f: E_{1} \rightarrow E_{2}$ is a map of $H$-comodules if the diagram

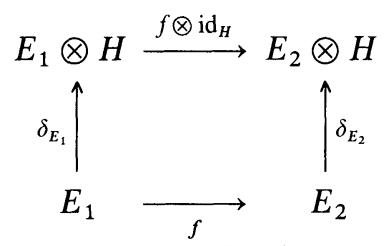

commutes. We could call such a map coequivariant or $H$-map. We denote the set of $H$-comodule maps by $\operatorname{Hom}_{H}\left(E_{1}, E_{2}\right)$. If $E_{1}$ and $E_{2}$ carry some additional structure (like being $k$-algebras) we mean by $\operatorname{Hom}_{H}\left(E_{1}, E_{2}\right)$ the subset of the morphisms of the appropriate category preserving the $H$-comodule structure.

2.2. $H$-Maps of $H$. We want to generalize the concept of a $G$-selfmap of $G$. $H$ itself is an $H$-comodule on the left and on the right, the diagrams defining the structure of $H$ yield immediately that the diagrams (1) commute, if we take $\Delta$ as the coaction. In the sequel, we will only use the right- $H$-coaction on $H$, writing $\operatorname{End}_{H}(H)$ for $H$-maps of $H$ is therefore unambiguous.

The "geometric" structure of $H$ is reflected in the algebra structure, so in requiring that $f$ also be a $k$-algebra homomorphism, we obtain a close analogue of a $G$-map $G \rightarrow G$.

Lemma 1. Let $f: H \rightarrow H$ be an $H$-map. Then $\xi:=\varepsilon \circ f: H \rightarrow k$ is a k-algebra homomorphism and $f$ can be written as $f=m_{k, H} \circ\left(\xi \otimes \mathrm{id}_{H}\right) \circ \Delta$, where $m_{k, H}: k \otimes H \rightarrow H$ denotes multiplication.

Proof. Since $\xi$ is a composition of homomorphisms of $k$-algebras, it is itself a homomorphism of $k$-algebras. We have to show that the diagram

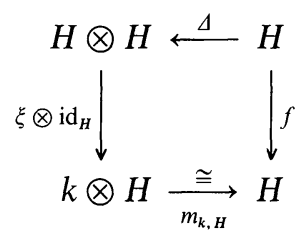

commutes. But this diagram is obtained by stacking the first of the following two diagrams on top of the second.

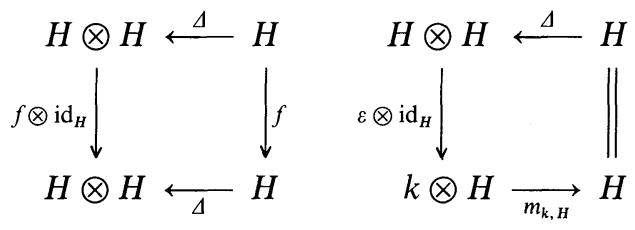


The first of them is commutative because $f$ is a map of $H$-comodules, the second is one of the axioms for the counit $\varepsilon$ [5].

The lemma justifies the following definition.

Definition 1. A $k$-algebra homomorphism $H \rightarrow k$ is called a character, the set of characters is denoted by $\mathbb{X}(H)$. The map $\chi: \operatorname{End}_{H}(H) \rightarrow \mathbb{X}(H): f \mapsto \varepsilon \circ f$ is called the character map.

The inverse of the character map sends $\xi \in \mathbb{X}(H)$ to $m_{k, H} \circ\left(\xi \otimes \operatorname{id}_{H}\right) \circ \Delta$. Hence characters correspond bijectively to $H$-maps of $H$, and $\mathbb{X}(H)$ inherits a composition from $\operatorname{End}_{H}(H)$ via $\chi$. If $f_{1}$ and $f_{2}$ are two $H$-maps of $H$, then their composition is also an $H$-map, the corresponding character $\xi$ can be computed using the previous lemma: $\xi=\varepsilon \circ f_{1} \circ f_{2}$. But if $\xi_{i}=\chi\left(f_{i}\right), i=1,2$, then

$$
\begin{aligned}
\xi & =\varepsilon \circ f_{1} \circ f_{2} \\
& =\varepsilon \circ m_{k, H} \circ\left(\xi_{1} \otimes \mathrm{id}_{H}\right) \circ \Delta \circ m_{k, H} \circ\left(\xi_{2} \otimes \mathrm{id}_{H}\right) \circ \Delta \\
& =\varepsilon \circ m_{H, k} \circ\left(m_{k, H} \otimes \mathrm{id}_{k}\right) \circ\left(\mathrm{id}_{k} \otimes \xi_{1} \otimes \mathrm{id}_{H}\right) \circ\left(\xi_{2} \otimes \mathrm{id}_{H \otimes H}\right) \circ\left(\mathrm{id}_{H} \otimes \Delta\right) \circ \Delta \\
& =m_{k} \circ\left(\mathrm{id}_{k} \otimes m_{k}\right) \circ\left(\xi_{2} \otimes \xi_{1} \otimes \varepsilon\right) \circ\left(\mathrm{id}_{H} \otimes \Delta\right) \circ \Delta \\
& =m_{k} \circ\left(m_{k} \otimes \mathrm{id}_{k}\right) \circ\left(\xi_{2} \otimes \xi_{1} \otimes \varepsilon\right) \circ\left(\Delta \otimes \mathrm{id}_{H}\right) \circ \Delta \\
& =m_{k} \circ\left(\left[m_{k} \circ\left(\xi_{2} \otimes \xi_{1}\right) \circ \Delta\right] \otimes \varepsilon\right) \circ \Delta \\
& =m_{k} \circ\left(\xi_{2} \otimes \xi_{1}\right) \circ \Delta \circ m_{H, k} \circ\left(\mathrm{id}_{H} \otimes \varepsilon\right) \circ \Delta \\
& =m_{k} \circ\left(\xi_{2} \otimes \xi_{1}\right) \circ \Delta .
\end{aligned}
$$

In the last step we used one of the axioms for the counit $\varepsilon$. With this composition, $\chi$ becomes a homomorphism of semigroups.

What we have done so far holds in any coalgebra. The interesting things begin to happen with the antipode $i$ :

Proposition 2. Every $H$-map $f: H \rightarrow H$ is invertible. If $\xi=\chi(f)$ is the character of $f$, then $\xi \circ i$ is the character of $f^{-1}: \xi \circ i=\chi\left(f^{-1}\right)$.

Proof. We have to show that $f_{1}:=f \circ m_{k, H} \circ\left(\xi \circ i \otimes \mathrm{id}_{H}\right) \circ \Delta=\mathrm{id}_{H}$ and $f_{2}:=m_{k, H} \circ\left(\xi \circ i \otimes \operatorname{id}_{H}\right) \circ \Delta \circ f=\operatorname{id}_{H}$. Since id ${ }_{H}$ has $\varepsilon$ as corresponding character, we only have to show that $\chi\left(f_{1}\right)=\varepsilon$ and $\chi\left(f_{2}\right)=\varepsilon$. We compute

$$
\begin{aligned}
\varepsilon \circ f_{1} & =\varepsilon \circ m_{k, H} \circ\left(\xi \otimes \operatorname{id}_{H}\right) \circ \Delta \circ m_{k, H} \circ\left(\xi \circ i \otimes \mathrm{id}_{H}\right) \circ \Delta \\
& =m_{k} \circ\left(\xi \otimes \mathrm{id}_{k}\right) \circ\left(\mathrm{id}_{H} \otimes \varepsilon\right) \circ \Delta \circ m_{k, H} \circ\left(\xi \circ i \otimes \mathrm{id}_{H}\right) \circ \Delta \\
& =\xi \circ m_{H, k^{\prime}} \circ\left(\mathrm{id}_{H} \otimes \varepsilon\right) \circ \Delta \circ m_{k, H} \circ\left(\xi \circ i \otimes \mathrm{id}_{H}\right) \circ \Delta \\
& =\xi \circ m_{k, H} \circ\left(\xi \circ i \otimes \mathrm{id}_{H}\right) \circ \Delta \\
& =m_{k} \circ(\xi \otimes \xi) \circ\left(i \otimes \mathrm{id}_{H}\right) \circ \Delta \\
& =\xi \circ m \circ\left(i \otimes \mathrm{id}_{H}\right) \circ \Delta \\
& =\xi \circ \eta \circ \varepsilon=\varepsilon .
\end{aligned}
$$

In the last step we use the fact that $\xi$ preserves the unit and is $k$-linear. The computation for $f_{2}$ is completely analogous. This completes the proof.' 
The character map is therefore not only a homomorphism of semigroups, but of groups. We can sum up the results of this section in the following corollary.

Corollary 3. $\operatorname{End}_{H}(H)$ is a group. The set $\mathbb{X}(H)$ with the maps

$$
\begin{aligned}
& \mu: \mathbb{X}(H) \times \mathbb{X}(H) \rightarrow \mathbb{X}(H):\left(\xi_{1}, \xi_{2}\right) \mapsto m_{k} \circ\left(\xi_{2} \otimes \xi_{1}\right) \circ \Delta \\
& \iota: \mathbb{X}(H) \rightarrow \mathbb{X}(H): \xi \mapsto \xi \circ i
\end{aligned}
$$

is a group. The map $\chi: \operatorname{End}_{H}(H) \rightarrow \mathbb{X}(H)$ is a group homomorphism.

2.3. The Character Group of a Quantum Group. Quantum groups in the sense of Woronowicz [8] are $C^{*}$-algebras, and the relevant maps have to satisfy some compatibility conditions with the $*$-structure. More precisely, $H$ is a $C^{*}$-algebra with unity and there are elements $u_{i k} \in H$ that generate a dense subalgebra $H^{0}$. There is a $C^{*}$-homomorphism $\Delta: H \rightarrow H \otimes H$, which satisfies

$$
\Delta\left(u_{k l}\right)=\sum_{r=1}^{n} u_{k r} \otimes u_{r l}
$$

Furthermore, there is a linear antimultiplicative mapping $i: H^{0} \rightarrow H^{0}$ such that $i\left(i\left(h^{*}\right)^{*}\right)=h$ for all $h \in H^{0}$, and a hermitian character $\varepsilon: H^{0} \rightarrow \mathbb{C}$ such that $H^{0}$, equipped with these maps, is a Hopf algebra (a hermitian character is a $C^{*}$ homomorphism). The character $\varepsilon$ satisfies $\varepsilon\left(u_{i k}\right)=\delta_{i k}$. The only obstruction for $H$ to be a Hopf algebra is that $i$ and $\varepsilon$ are not defined on the whole of $H$.

In some cases $\varepsilon$ can not be extended to the whole of $H$, and then $\varepsilon \circ f$ might not be well defined for some $C^{*}$-homomorphisms which respect the $H$-coaction. It is therefore necessary to restrict the concept of $H$-map to $C^{*}$-homomorphisms which preserve $H^{0}$ :

Definition 2. Let $H$ be a quantum group, and $f: H \rightarrow H$ a $C^{*}$-homomorphism such that $f\left(H^{0}\right) \subset H^{0}$ and the diagrams (1) with $E=H$ and $H$ replaced by $H^{0}$ at appropriate places commute. Such an $f$ is called an $H$-map of $H$. The set of $H$-maps of $H$ is again denoted by $\operatorname{End}_{H}(H)$.

So we are only interested in maps which induce a $H^{0}$-map of $H^{0}$, and for these we can apply the results of the preceding section. In particular, every $H^{0}$-map is an isomorphism $H^{0} \rightarrow H^{0}$, and every character of an $H^{0}$-map is hermitian. For our purposes, only hermitian characters are interesting, we therefore define $\mathbb{X}(H)$ to contain only hermitian characters, and we will have to say more about continuity in a moment.

$H^{0}$ is very special, it is generated by the elements $u_{i k} \in H$, the character associated to an $H$-map $f$ is therefore uniquely determined by the values on these elements. Furthermore, the composition of Corollary 3 can also be expressed by these values of the character. Let $\xi_{1}$ and $\xi_{2}$ be two characters of $H^{0}$, then their composition $\xi=\xi_{1} \cdot \xi_{2}$ has the following value on $u_{i k}$ :

$$
\begin{aligned}
\xi\left(u_{i k}\right) & =m_{\mathbb{C}} \circ\left(\xi_{2} \otimes \xi_{1}\right) \circ \Delta\left(u_{i k}\right) \\
& =m_{\mathbb{C}} \circ\left(\xi_{2} \otimes \xi_{1}\right) \sum_{j=1}^{n} u_{i j} \otimes u_{j k} \\
& =\sum_{j=1}^{n} \xi_{2}\left(u_{i j}\right) \xi_{1}\left(u_{j k}\right) .
\end{aligned}
$$


But this is almost matrix multiplication. Let us define

$$
\varphi: \mathbb{X}\left(H^{0}\right) \rightarrow M_{n}(\mathbb{C}): \xi \mapsto\left(x_{i k}\right):=\left(\xi\left(u_{i k}\right)\right)^{t} .
$$

This map respects the composition; it turns $\xi_{1} \cdot \xi_{2}$ into the matrix product of $\varphi\left(\xi_{1}\right)$ and $\varphi\left(\xi_{2}\right)$. Since the set of characters is a group, $\operatorname{im} \varphi$ is a subgroup of $G L_{n}(\mathbb{C})$, or in other words, $\varphi$ is a group homomorphism, and we know that it is injective.

The problem with all this is that $\varepsilon$ might not be continuous. This means that we cannot expect $\chi(f)$ to be continuous either, although $f$ might be. In many interesting cases of quantum groups, $\varepsilon$ is continuous, for instance for $S U_{\mu}(n)$ discussed in Chapter 5. Thus we reach our final definition of $\mathbb{X}(H)$ :

Definition 3. If $H$ is a quantum group, then $\mathbb{X}(H)$ is the set of continuous hermitian characters of $H$, i.e. unital $C^{*}$-homomorphisms $H \rightarrow \mathbb{C}$.

But even for $\xi \in \mathbb{X}(H)$ it is not entirely clear that the candidate for the character of the inverse, $\xi \circ i$, which is only defined on $H^{0}$ a priori, can be continuously extended to $H$ and thus gives an $H$-map. That such a thing can not happen is shown in the next proposition. If $\varepsilon$ is continuous, then $\mathbb{X}(H)$ is nonempty, the next proposition also gives some kind of a converse to this statement.

Proposition 4. Let $H$ be a quantum group such that $\mathbb{X}(H)$ is nonempty. Then $\mathbb{X}(H)$ is in a canonical way a compact Lie group.

Proof. The idea of the proof is as follows: we first show that $\mathbb{X}(H)$ is a compact subset of $G L_{n}(\mathbb{C})$, closed under multiplication. Then we show that such a set is automatically a group, contained in some compact Lie group. The Lie group structure then follows from some standard result of the theory of compact Lie groups.

Since $H$ contains a finitely generated dense subalgebra, it is separable. By $[2,15.3 .2]$, the set $\mathbb{X}(H)$ is compact in the topology induced from the weak topology of the dual of $H$. In particular, the maps $\xi \mapsto \xi\left(u_{i k}\right)$ are continuous, hence the image of $\mathbb{X}(H)$ in $G L_{n}(\mathbb{C})$ under the map $\varphi$ is compact. $\varphi: \mathbb{X}(H) \rightarrow G L_{n}(\mathbb{C})$ is even a homeomorphism onto the image, because any continuous injection from a compact set has this property. Since the definition of the composition of characters only involves the $C^{*}$-homomorphism $\Delta, \mathbb{X}(H)$ is closed under composition, hence also its image in $G L_{n}(\mathbb{C})$.

Next we show that any compact subset $K \subset G L_{n}(\mathbb{C})$ closed under multiplication is a subgroup. If $K$ does not contain an element other than the identity, then $K$ is trivially a subgroup. Therefore let $k \in K$ be a nontrivial element and consider the set $A=\left\{k^{r} \mid r>0\right\} \subset K$.

There is an element $g \in G L_{n}(\mathbb{C})$, such that $g^{-1} k g$ has Jordan normal form, i.e. it is decomposed into blocks of the form

$$
m=\left(\begin{array}{llll}
\lambda & 1 & & \\
& \lambda & \ddots & \\
& & \ddots & 1 \\
& & & \lambda
\end{array}\right) .
$$

The powers of $m$ have $\lambda^{r}$ on the diagonal. For $|\lambda|>1$ this implies that the set $A \subset M_{n}(\mathbb{C}) \subset \mathbb{C}^{n \times n}$ is unbounded, hence cannot be contained in a compact set. If 
$|\lambda|<1$ then $k^{-1}$ has a block like $m$ with $\lambda$ replaced by $\lambda^{-1}$. The same argument as before then shows that $\left\{k^{-r} \mid r>0\right\}$ cannot be contained in the compact set $K^{-1}$. Hence $|\lambda|=1$.

The matrix element $\left(m^{r}\right)_{12}$ is $r \lambda^{r-1}$, which is again unbounded: $\left|r \lambda^{r-1}\right|=r$. This shows that only $1 \times 1$ Jordan blocks are possible, or that $k$ is contained in a conjugate of the torus $T=\left\{\operatorname{diag}\left(\lambda_{1}, \ldots, \lambda_{n}\right)|| \lambda_{i} \mid=1 \forall i\right\}$. There are two situations: either $A$ is a finite set, but then it is trivially a cyclic group, or $A$ is infinite, but then it is dense in some closed abelian subgroup of $T$. But $K \cap T$ is closed, so $\bar{A} \subset K \cap T$ is a subgroup of $T$, which means that the identity and the inverse of $k$ are both in $K$, hence $K$ is a closed subgroup of $G L_{n}(\mathbb{C})$.

$K$ is a compact topological group equipped with a faithful representation, the injection $\varphi$ of $K$ into $G L_{n}(\mathbb{C})$. By the unitarian trick, we can conjugate $K$ into $U(n)$; we simply have to change the basis in such a way as to make the representation unitary with respect to the standard inner product. But any closed subgroup of a compact Lie group is a Lie subgroup [1].

In the rest of this paper, we only consider quantum groups with the property alluded to in the proposition:

Definition 4. A quantum group $H$ is called grouplike, if $\mathbb{X}(H)$ is nonempty.

2.4. Associated Representations of the Character Group. Let $H$ be a group-like quantum group. Recall that $\varrho \in B(K) \otimes H$ is called a representation of $H$ in the Hilbert space $K$ if

$$
\left(\mathrm{id}_{B(K)} \otimes \Delta\right)(\varrho)=\left(m_{B(K)} \otimes \mathrm{id}_{H \otimes H}\right) \circ S_{(23)} \circ(\varrho \otimes \varrho),
$$

where $S_{(23)}$ is the map that exchanges the second and third factor in the tensor product, and $m_{B(K)}$ is the multiplication in $B(K)$. A representation $\varrho$ of $H$ induces in a natural way a representation of the character group. We denote this new representation again by $\varrho$. Its value on $\xi \in \mathbb{X}(H)$ is the operator $\left(\operatorname{id}_{B(K)} \otimes \xi\right)(\varrho) \in B(K)$. We verify the homomorphism property $\varrho\left(\xi_{1}\right) \varrho\left(\xi_{2}\right)=$ $\varrho\left(\xi_{1} \xi_{2}\right)$ :

$$
\begin{aligned}
\varrho\left(\xi_{1} \xi_{2}\right) & =\varrho\left(m_{\mathbb{C}} \circ\left(\xi_{1} \otimes \xi_{2}\right) \circ \Delta\right) \\
& =\left(\operatorname{id}_{B(K)} \otimes\left[m_{\mathbb{C}} \circ\left(\xi_{1} \otimes \xi_{2}\right) \circ \Delta\right]\right)(\varrho) \\
& =\left(\operatorname{id}_{B(K)} \otimes \xi_{1} \otimes \xi_{2}\right) \circ\left(\operatorname{id}_{B(K)} \otimes \Delta\right)(\varrho) \\
& =m_{B(K)}\left(\left(\operatorname{id}_{B(K)} \otimes \xi_{1}\right)(\varrho) \otimes\left(\operatorname{id}_{B(K)} \otimes \xi_{2}\right)(\varrho)\right) \\
& =\varrho\left(\xi_{1}\right) \varrho\left(\xi_{2}\right) .
\end{aligned}
$$

Note that this is essentially the proof for the homomorphism property of $\varphi$; this is not surprising, the matrix elements $u_{i k}$ form a representation $u \in M_{n}(\mathbb{C}) \otimes H$.

\section{Deformations of Quantum Groups and Their Character Groups}

Compact Lie groups are very rigid, one cannot deform them. Quantum groups however allow much more flexibility; there are deformations of quantum groups and associated deformations of their character groups. All quantum groups in this and the following chapters are grouplike. 
3.1. Deformations of Quantum Groups. Since our quantum groups are $C^{*}$-algebras, a natural notion of deformation is that of a continuous field of $C^{*}$-algebras with the additional properties that the fibres are quantum groups, the relevant structure maps are continuous and the matrix elements $u_{i k}$ are continuous sections.

In this and the following sections, let $X$ be a path connected and simply connected topological space, the parameter space. We consider families $\mathscr{F}=\left(\mathscr{F}_{x}\right)_{x \in X}$ of objects in some subcategory of the category of sets, and a map $f: X \rightarrow \coprod_{x \in X} \mathscr{F}_{x}$ is called a section if $f(x) \in \mathscr{F}_{x}$ for all $s \in X$. We write $Y$ for the trivial family with constant fibre $Y$, for this family $\coprod_{x \in X} Y_{x}=X \times Y$.

Recall [3] that a family of $C^{*}$-algebras $\mathscr{A}=\left(\left(A_{x}\right)_{x \in X}, \Gamma_{\mathscr{A}}\right)$ is called a continuous field of $C^{*}$-algebras, if the set of sections $\Gamma_{\mathscr{A}}$ satisfies

(1) $\Gamma_{\mathscr{A}}$ is closed under addition, the $*$-operation, pointwise multiplication and multiplication by continuous functions on $X$.

(2) $x \mapsto\|a(x)\|$ is continuous for all $a \in \Gamma_{\mathscr{A}}$.

(3) $\left\{s(x) \mid s \in \Gamma_{\mathscr{A}}\right\}$ is dense in $A_{x}$.

A map between two continuous fields $\mathscr{A}_{1} \rightarrow \mathscr{A}_{2}$ is a family of continuous maps $f_{x}: A_{1, x} \rightarrow A_{2, x}$ such that $x \mapsto f_{x} \circ S(x)$ is a continuous section of $\mathscr{A}_{2}$ for every continuous section $s \in \Gamma_{\mathscr{A}_{1}}$.

A continuous field of quantum groups is a continuous field of $C^{*}$-algebras $\mathscr{H}$, together with sections $u_{i k} \in \Gamma_{\mathscr{H}}$ that generate $\Gamma_{\mathscr{H}}$ as a $C(X)$-algebra. The $u_{i k}$ generate in every fibre a dense subalgebra $H_{x}^{0}$. Furthermore we have maps

$$
\begin{aligned}
& \Delta: \mathscr{H} \rightarrow \mathscr{H} \otimes \mathscr{H} \\
& \varepsilon: \mathscr{H}^{0} \rightarrow \mathbb{C} \\
& i: \mathscr{H}^{0} \rightarrow \mathscr{H}^{0}
\end{aligned}
$$

such that $\left(H_{x}, \Delta_{x}, \varepsilon_{x}, i_{x}, u_{i k}(x), 1 \leqq i, k \leqq n\right)$ is a quantum group for every $x \in X$. Although $i$ is not defined on the whole of the fibres, it makes sense to speak of continuity of the field $i, i$ is only supposed to compose nicely with sections on a subalgebra where it is well defined. Note that $i \circ s$ may not be continuous if $s$ is not in this subalgebra.

An example of a continuous field of quantum groups is given by the twisted $S U_{\mu}(n)$ groups of Woronowicz [9]. This example is studied in more detail in Chap. 5.

Almost everything we did for quantum groups has a more or less direct translation to fields of quantum groups. We only have to interpret the defining commutative diagrams of Chap. 2 fibrewise, and all the structure maps have to be continuous fields. For instance there are fields of Banach algebras which are at the same time $\mathscr{H}$-comodules on the right. Such a field $\mathscr{E}$ has as fibre $E_{x}$ over the point $x$ an $H_{x}$-comodule. Furthermore the coaction map $\delta_{\mathscr{E}}$ has to be a continuous field of coaction maps. We use the notation $\operatorname{Hom}_{\mathscr{H}}\left(\mathscr{E}_{1}, \mathscr{E}_{2}\right)$ for the $\mathscr{H}$-maps between $\mathscr{H}$-comodules $\mathscr{E}_{1}$ and $\mathscr{E}_{2}$. Also $\mathscr{H}$ itself is a $\mathscr{H}$-comodule on the right, and we mean this coaction when we write $\operatorname{End}_{\mathscr{H}}(\mathscr{H})$.

As before, the elements of $\operatorname{End}_{\mathscr{H}}(\mathscr{H})$ are in bijective correspondence with the characters, characters are now continuous fields of $*$-homomorphisms from $\mathscr{H}$ to the trivial field $\mathbb{C}$. 
3.2. The Family of Character Groups. Instead of just one character group we have now a whole family $\mathbb{X}\left(H_{x}\right)$ of groups, indexed by the points $x \in X$. There is a natural injection

$$
\varphi: \coprod_{x \in X} \mathbb{X}\left(H_{x}\right) \hookrightarrow X \times G L_{n}(\mathbb{C}):\left.\varphi\right|_{\mathbb{X}\left(H_{x}\right)}=\varphi_{x}: \mathbb{X}\left(H_{x}\right) \hookrightarrow G L_{n}(\mathbb{C}) .
$$

There is a topology on $\coprod_{x \in X} \mathbb{X}\left(H_{x}\right)$, generated by the following open sets. The character groups are subsets of the duals $H_{x}^{\prime}$, which form a continuous field $\mathscr{H}^{\prime}$ of Banach spaces on $X$. Let $s$ be a section of $\mathscr{H}^{\prime}$ such that $s(x) \in \mathbb{X}\left(H_{x}\right)$ for all $x$. For any open subset $W$ of $X$ and any $\varepsilon>0$, let

$$
U(s, W, \varepsilon)=\left\{b \in \coprod_{x \in W} H_{x}^{\prime} \mid b \in H_{x}^{\prime} \Rightarrow\|b-s(x)\|<\varepsilon\right\} \cap \coprod_{x \in X} \mathbb{X}\left(H_{x}\right) .
$$

The topology generated by these open sets is the same as the relative topology of the image of $\varphi$ in $X \times G L_{n}(\mathbb{C})$, so $\varphi$ is a homeomorphism onto the image.

Definition 5. $\mathbb{X}(\mathscr{H})$ is the disjoint union of the $\mathbb{X}\left(H_{x}\right)$ equipped with the above topology. Let $\mathrm{pr}_{1}$ be the projection $\mathbb{X}(\mathscr{H}) \rightarrow X$ which maps $\mathbb{X}\left(H_{x}\right)$ to $x$.

Note that there is no relationship to the topology on the product $\prod_{x \in X} \mathbb{X}\left(\mathscr{H}_{x}\right)$. The product is the set of all the sections of $\mathbb{X}(\mathscr{H})$, but we are only interested in those sections which are continuous maps $X \rightarrow \mathbb{X}(\mathscr{H})$.

If $f: \mathscr{H} \rightarrow \mathscr{H}$ is an $\mathscr{H}$-map, then $\chi(f)=\varepsilon \circ f$ is a continuous field of characters, i.e. a continuous map $X \rightarrow \mathbb{X}(\mathscr{H})$. We conjecture of course that an analogue to Proposition 2 is true.

Proposition 5. Every $\mathscr{H}$-map of $\mathscr{H}$ is an isomorphism, the character of the inverse has $\chi(f) \circ i$ as its restriction to $\mathscr{H}^{0}$.

Proof. Let $f: \mathscr{H} \rightarrow \mathscr{H}$ be an $\mathscr{H}$-map, i.e. a homomorphism of continuous fields of $C^{*}$-algebras and of right- $\mathscr{H}$-comodules. Then the character map $\chi$ associates to $f$ the map $\varepsilon \circ f$, which is a continuous map $X \rightarrow \coprod_{x \in X} \mathbb{X}\left(H_{x}\right)$. Since $i$ is a continuous family of antipodes, $\chi(f) \circ i$ is also continuous, it is therefore the character of a $\mathscr{H}$-map. It is the inverse to $f$.

3.3. Deformations of Representations. Let $K$ be a Hilbert space. A deformation of a quantum group representation is a continuous section $\varrho$ of the field

$$
B(K) \otimes \mathscr{H}=\left(\left(B(K) \otimes H_{x}\right)_{x \in X}, B(K) \otimes \Gamma_{\mathscr{H}}\right)
$$

with the property that $\varrho_{x}$ is a representation of $H_{x}$ for every $x \in X$. There is a family of representations of $\mathbb{X}\left(H_{x}\right)$ associated to $\varrho$, denoted by the same symbol and mapping $\xi \in \mathbb{X}\left(H_{x}\right)$ to the operator $\left(\operatorname{id}_{B(K)} \otimes \xi\right)\left(\varrho_{x}\right)$.

\section{Structure Groups and Principal Bundles}

A principal fibre bundle is locally completely characterized by its structure group $G$. Over some suitably small open set $U$ in the base space, the bundle looks like the trivial bundle $U \times G$. Its sections are therefore the same as the elements of the group map $(U, G)$, which we will henceforth also call structure group. Hence a principal 
bundle is nothing else but chunks of the structure group glued together in some unorthodox way destroying the group structure in the process and only keeping the group action intact.

For this chapter we fix a continuous field of quantum groups $\mathscr{H}$ over some path connected, simply connected and locally contractible parameter space $X$. A path connected and simply connected manifold (with or without boundary) has these properties.

4.1. Structure Groups. Let $M$ be a compact topological space, and $g: M \rightarrow X$ a continuous map. Then there exists a canonically defined continuous field $g^{*} \mathscr{H}$ of quantum groups on $M$. The fibre over $m \in M$ is $\mathscr{H}_{g(m)}$, the set of sections $\Gamma_{g^{*} \mathscr{H}}$ is the $C(M)$-subalgebra of $\prod_{m \in M} H_{g(m)}$ generated by the pullbacks $\left(g^{*} s\right)(m):=s(g(m))$ of sections $s \in \Gamma_{\mathscr{H}}$. The verification of the axioms is immediate. This field of quantum groups is called the structure group associated to $g$. To $\mathscr{G}=g^{*} \mathscr{H}$ we can apply all the results of the previous chapter.

The construction is of course much more general, to every continuous field of $C^{*}$-algebras on $X$ and to every continuous map $M \rightarrow X$ we can associate a pullback. Pullbacks to embeddings of subspaces are called restrictions.

The classical case is recovered for $X=\{\mathrm{pt}$.$\} , then there is only one quantum$ group to choose from, and the structure group is simply the trivial bundle $M \times G$. The term is justified because the continuous sections of the bundle form a group.

The virtue in fixing a parameter space $X$ is that the class of possible fields of quantum groups is somewhat limited. Also the way in which the quantum groups are allowed to vary along a path in the base space $M$ is restricted to the ways they can vary along a path in $X$. Later we will have to restrict $X$ further.

Any closed subspace of $M$ gets a field of quantum groups from $M$ by restriction; it is the pullback of $\mathscr{H}$ under the restriction of $f$ to the subspace. If $f_{0}^{*} \mathscr{H}$ and $f_{1}^{*} \mathscr{H}$ are two structure groups, then a map $F: M \times I \rightarrow X(I$ is the interval $[0,1])$ such that $\left.F\right|_{M \times\{0\}}=f_{0}$ and $\left.F\right|_{M \times\{1\}}=f_{1}$ is called a homotopy of the structure groups. It induces a structure group $F^{*} \mathscr{H}$ on $M \times I$ such that its restrictions to $M \times\{0\}$ and $M \times\{1\}$ are $f_{0}^{*} \mathscr{H}$ and $f_{1}^{*} \mathscr{H}$ respectively.

A representation $\varrho$ of $\mathscr{H}$ can also be pulled back to $g^{*} \mathscr{H}$, as well as the family of character groups.

4.2. Principal Bundles. Let $\mathscr{G}=g^{*} \mathscr{H}$ be a structure group on $M$, then a $\mathscr{G}$-bundle $\mathscr{P}$ is a continuous field of $C^{*}$-algebras, such that every fibre $P_{m}$ is a $G_{m}$-comodule isomorphic as a $C^{*}$-algebra to $G_{m}$. It is locally trivial if there is a covering of $M$ by open sets $U_{\alpha}, \alpha$ ranging over some indexing set, such that for every restriction $\left.\mathscr{P}\right|_{U_{\alpha}}$ there is a $\mathscr{G}_{U_{\alpha}}$-isomorphism

$$
\varphi_{\alpha}:\left.\mathscr{P}\right|_{\left.U_{\alpha} \rightarrow \mathscr{G}\right|_{U_{\alpha}},}
$$

such a map is as usual called a bundle chart. Since $\varphi_{\alpha}$ is invertible, we get maps on the intersection $U_{\alpha} \cap U_{\beta}$ :

$$
\varphi_{\beta \alpha}:\left.\left.\mathscr{G}\right|_{U_{\alpha} \cap U_{\beta}} \rightarrow \mathscr{G}\right|_{U_{\alpha} \cap U_{\beta}}, \quad \varphi_{\beta \alpha}=\varphi_{\beta}{ }^{\circ} \varphi_{\alpha}^{-1} .
$$

Specifying these maps allows to reconstruct the bundle $\mathscr{P}$ completely because of the following proposition which is useful in its own.

Proposition 6. Let $f: \mathscr{P}_{1} \rightarrow \mathscr{P}_{2}$ be a $\mathscr{G}$-map of principal $\mathscr{G}$-bundles. Then $f$ is an isomorphism of principal $\mathscr{G}$-bundles. 
Proof. The only thing we have to prove is that the inverse of $f$ depends continuously on $m \in M$. This question is purely local, we can therefore work in an open set $U \subset M$ over which both bundles are trivial, $f$ then induces a $\left.\mathscr{G}\right|_{U}$-map of $\left.\mathscr{G}\right|_{U}$. After restriction to $U$ we get the situation of Proposition 5, which completes the proof.

We would not only like to consider bundles with some fixed structure group, but also bundles with different structure groups corresponding to different maps $f: M \rightarrow X$.

Definition 6. $\mathscr{P}$ is called a $\mathscr{H}$-bundle if it is a $f^{*} \mathscr{H}$-bundle for some continuous $f: M \rightarrow X$.

There are two special classes of $\mathscr{H}$-bundles. A bundle globally isomorphic to a structure group is called a trivial bundle; a trivial bundle with structure group induced by a constant map is called a constant $\mathscr{H}$-bundle.

The bundles in an isomorphism class of principal bundles have all the same structure group; they do not take the flexibility of quantum groups into account. The set of isomorphism classes of $\mathscr{H}$ bundles on $M$ is therefore very large in general. There are roughly as many trivial bundles as there are continuous maps $M \rightarrow X$. Isomorphism is much too strong a relation.

As we were able to construct homotopies of the structure group, we can also consider homotopies of principal bundles.

Definition 7. $\mathscr{P}_{0}$ and $\mathscr{P}_{1}$ are called homotopic, if there is a bundle $\mathscr{P}$ on $M \times I$ with structure group $G^{*} \mathscr{H}$, such that $\mathscr{P}_{0}=\left.\mathscr{P}\right|_{M \times\{0\}}$ and $\mathscr{P}_{1}=\left.\mathscr{P}\right|_{M \times\{1\}}$ which have structure group $\left(\left.G\right|_{M \times\{0\}}\right)^{*} \mathscr{H}$ and $\left(\left.G\right|_{M \times\{1\}}\right)^{*} \mathscr{H}$ respectively. $\mathscr{P}$ is called a homotopy between $\mathscr{P}_{0}$ and $\mathscr{P}_{1}$, also written $\mathscr{P}: \mathscr{P}_{0} \simeq \mathscr{P}_{1}$.

The following lemma is immediately verified.

Lemma 7. Homotopy of principal bundles is an equivalence relation.

We want to study the set of homotopy classes of bundles over a given space $M$, which we denote by $\operatorname{Bund}(M, \mathscr{H})$. In the classical case, this amounts to finding all the free homotopy classes of maps from $M$ into $B G$. To prove this, one first introduces a basepoint and later studies the effect of changing the base point.

From now on we work in $\mathrm{CW}_{*}$, the homotopy category of path connected pointed CW-complexes. We start by introducing basepoints in our situation. First of all, the parameter space $X$ has now a basepoint, which means that one of the quantum groups is preferred. The base spaces are now in fact pairs $(M, *)$. A bundle over the pair $(A, B)$ is of course a pair of bundles $\left(\mathscr{P}_{A}, \mathscr{P}_{B}\right)$ over the individual spaces together with an isomorphism of bundles $\left.\mathscr{P}_{B} \cong \mathscr{P}_{A}\right|_{B}$. For a pointed space this means that a bundle is now naturally equipped with an isomorphism $P_{*} \cong H_{*}$. Also homotopies are now supposed to preserve the basepoint. Unless explicitly stated, all maps are supposed to preserve the additional structure.

We can now define a contravariant functor on $\mathrm{CW}_{*}$ which assigns to a space $M$ the set of homotopy classes of $\mathscr{H}$-bundles on $M$ and to a map $f: M_{1} \rightarrow M_{2}$ and a bundle on $M_{2}$ its pullback. If $\mathscr{P}$ is a homotopy of the bundles $\mathscr{P}_{0}$ and $\mathscr{P}_{1}$ on $M_{2}$, then $\left(f \times \mathrm{id}_{I}\right)^{*} \mathscr{P}$ is a homotopy of the bundles $f^{*} \mathscr{P}_{0}$ and $f^{*} \mathscr{P}_{1}$. If $F: f_{0} \simeq f_{1}$ is a homotopy, then $F^{*} \mathscr{P}$ is a homotopy of the bundles $f_{0}^{*} \mathscr{P}$ and $f_{1}^{*} \mathscr{P}$ on $M_{1}$ for any bundle $\mathscr{P}$ on $M_{2}$. 
Because we assumed that $X$ is path connected, and because trivial bundles with constant fibres correspond to constant maps into $X$, all the bundles with constant fibres are homotopic, they define a base point in the set of homotopy classes of bundles. Since $X$ is assumed to be simply connected, trivial bundles over a circle $S^{1}$ are homotopic to a constant bundle. We summarize this in the following lemma about $\operatorname{Bund}_{*}(\cdot, \mathscr{H})$, analogous statements apply of course for Bund $(\cdot, \mathscr{H})$.

Lemma 8. Let $\operatorname{Bund}_{*}(M ; \mathscr{H})=\operatorname{Bund}_{*}(M)$ be the pointed set of homotopy classes of (pointed) $\mathscr{H}$-bundles, the basepoint being the class of a trivial bundle with constant structure group. For $f: M_{1} \rightarrow M_{2}, \operatorname{Bund}_{*}(f ; \mathscr{H})=\operatorname{Bund}_{*}(f)$ is the map that assigns to a bundle on $M_{2}$ its pullback to $M_{1}$. Bund ${ }_{*}$ is a contravariant functor from $\mathrm{CW}_{*}$ to the category of pointed sets, Bund ${ }_{*}: \mathrm{CW}_{*} \rightarrow$ Sets $_{*}$.

The homotopy relation allows a lot of flexibility:

Lemma 9. Let $\mathscr{P}$ be a bundle over $M$ and $m_{0} \in M$. Then there is a homotopic bundle with constant structure group over a sufficiently small neighborhood of $m_{0}$.

Proof. First take a trivializing covering for $\mathscr{P}$ and let $U_{0}$ be an open set of the covering which contains $m_{0}$. Let $u$ be a continuous function on $M$ with support in $U_{0}$ and $u\left(m_{0}\right)=1$. Then $V:=\left\{m \in M \mid u(m)>\frac{1}{2}\right\}$ is an open neighborhood of $m_{0}$ such that its closure $K$ is still contained in $U_{0}$. Replacing all other charts by $U_{\alpha} \backslash K$ gives an atlas where only one chart meets $V$.

Let $f^{*} \mathscr{H}$ be the structure group of $\mathscr{P}$. By making $V$ smaller if necessary, we can achieve that $V$ is mapped into a contractible neighborhood $W$ of $f\left(m_{0}\right)$. Let $\Phi_{t}: W \rightarrow W$ be a homotopy relative to $f\left(m_{0}\right)$ from the identity to the constant map. Let $u$ be a function $M \rightarrow I$ with support in $V$ and $u=1$ on some neighborhood of $m_{0}$. Then we change the structure group in $V$ by the homotopy $\Phi_{t u(m)}^{*} \mathscr{H}$; because $V$ does not meet any other chart besides $U_{0}$, this does not affect the coordinate changes and therefore defines a homotopy of the bundle $\mathscr{P}$ to one with the required properties.

4.3. Associated Vectorbundles. If $\varrho$ is a representation of $\mathscr{H}$ in the Hilbert space $K$, then to every principal $\mathscr{G}$-bundle we can associate in a canonical way a vectorbundle. We choose a covering of $M$ which trivializes the bundle $\mathscr{P}$. On every intersection of two open sets $U_{\alpha}$ and $U_{\beta}$ of the covering, we have a glueing map $\varphi_{\beta \alpha}$. This is a $\left.\mathscr{G}\right|_{U_{\alpha} \cap U_{\beta}}$-map of $\left.\mathscr{G}\right|_{U_{\alpha} \cap U_{\beta}}$, which is completely characterized by its character. The representation of $\mathscr{H}$ induces a family of representations of the character groups. To $\varphi_{\beta \alpha}$ are therefore associated continuous maps $u \rightarrow G L(K)$, and they can be used to glue together trivial $K$-bundles over the $U_{\alpha}$ to a $K$-bundle over $M$.

This construction is completely analogous to the construction of associated vectorbundles for $G$-bundles where $G$ is a group.

4.4. Classifying Spaces. We have constructed in Sect. 4.2 a functor from the category $\mathrm{CW}_{*}$ to the category of pointed sets. The existence of a classifying space for $\mathscr{H}$-bundles on pointed $\mathrm{CW}$-complexes follows from a general theorem of algebraic topology. This theorem says that the restriction of a functor $H$ on the homotopy category of pointed spaces to the subcategory of $\mathrm{CW}$-complexes is 
representable by a CW-space if and only if it is a homotopy functor $[7,7.7]$. A homotopy functor is a functor $H$ on $\mathrm{CW}_{*}$ such that

(a) If $j: Y \rightarrow Z$ is an equalizer of $f_{0}, f_{1}: A \rightarrow Y$, and if $u \in H(Y)$ is such that $H\left(f_{0}\right) u=H\left(f_{1}\right) u$, there is $v \in H(Z)$ such that $H(j) v=u$.

(b) If $\left(Y_{\lambda}\right)_{\lambda \in \Lambda}$ is an indexed family of objects of $\mathrm{CW}_{*}$ and $i_{\lambda}: Y_{\lambda} \rightarrow \bigvee_{\lambda \in \Lambda} Y_{\lambda}$, there is an equivalence

$$
\left(H\left(i_{\lambda}\right)\right)_{\lambda \in \Lambda}: H\left(\bigvee_{\lambda \in \Lambda} Y_{\lambda}\right) \cong \prod_{\lambda \in \Lambda} H\left(Y_{\lambda}\right) .
$$

Recall that an equalizer of $f_{0}, f_{1}: A \rightarrow Y$ is a morphism $j: Y \rightarrow Z$ such that $j \circ f_{0}=j \circ f_{1}$ and that for every other morphism $j^{\prime}: Y \rightarrow Z^{\prime}$ with this property, there is a morphism $h: Z \rightarrow Z^{\prime}$ such that $h \circ j=j^{\prime}$. In the category of CW-complexes, there are equalizers of the form $Z=Y \cup(A \times I)$ with the identifications $(a, 0) \sim f_{0}(a)$ and $(a, 1) \sim f_{1}(a)$ for all $a \in A$. The next lemma shows that it suffices to prove property (a) for these equalizers.

Lemma 10. Let $j: Y \rightarrow Z$ and $j^{\prime}: Y \rightarrow Z^{\prime}$ be two equalizers of $f_{0}, f_{1}: A \rightarrow Y$. If one of them satisfies (a), then so does the other.

Proof. Suppose $j: Y \rightarrow Z$ satisfies (a). Then there is $v \in H(Z)$ such that $H(j) v=u$. Since $j$ and $j^{\prime}$ are both equalizers, there is a map $h: Z^{\prime} \rightarrow Z$ such that $h \circ j^{\prime}=j$. Then $u=H(j) v=H\left(h \circ j^{\prime}\right)=h\left(j^{\prime}\right) \circ H(h) v$, and $v^{\prime}=H(h) v \in H\left(Z^{\prime}\right)$ is the element we are looking for: it satisfies $H\left(j^{\prime}\right) v^{\prime}=u$, hence $j^{\prime}$ satisfies (a).

We now return to the $\mathscr{H}$-bundle functor.

Proposition 11. The functor Bund $_{*}$ of $\mathscr{H}$-bundles satisfies (a).

Proof. Let $Z$ be the special equalizer exhibited above. Let $\mathscr{P}$ be a bundle on $Y$ such that $f_{0}^{*} \mathscr{P} \simeq f_{1}^{*} \mathscr{P}$, i.e. there is a bundle $\mathscr{Q}$ on $A \times\left[\frac{1}{3}, \frac{2}{3}\right]$ such that $\left.\mathscr{Q}\right|_{A \times\left\{\frac{1}{3}\right\}}=f_{0}^{*} \mathscr{P}$ and $\left.\mathscr{2}\right|_{A \times\left\{\frac{2}{3}\right\}}=f_{1}^{*} \mathscr{P}$. The compositions $f_{0} \circ \mathrm{pr}_{1}: A \times\left[0, \frac{1}{3}\right] \rightarrow Y$ and $f_{1} \circ \operatorname{pr}_{2}: A \times\left[\frac{2}{3}, 1\right]$ $\rightarrow Y$ induce bundles by pullback which can be glued together to a bundle on the subset $\left(A \times\left[\frac{2}{3}, 1\right] \cup Y \cup A \times\left[0, \frac{1}{3}\right]\right)$ of $Z$. The bundle $\mathscr{Q}$ fits exactly into the gap $A \times\left[\frac{1}{3}, \frac{2}{3}\right]$ to give a bundle $\mathscr{R}$ on $Z$. The restriction of $\mathscr{R}$ to $Y$ is of course $\mathscr{P}$, which proves property (a).

If Bund ${ }_{*}$ is to be a homotopy functor, then it should also satisfy (b). (b) says that it is possible to glue together bundles over the individual spaces $Y_{\lambda}$ to a global bundle on the wedge. Since we are in a pointed situation, there is no problem in glueing together bundles over a basepoint. The only way to glue the bundles together without destroying the additional structure is to use the canonical isomorphisms $\left.\mathscr{P}_{i}\right|_{\{*\}} \simeq \mathscr{H}_{*}$.

In this way we have constructed an inverse of

$$
\operatorname{Bund}_{*}\left(i_{\lambda}\right)_{\lambda \in \Lambda}: \operatorname{Bund}_{*}\left(\bigvee_{\lambda \in \Lambda} Y_{\lambda}\right) \rightarrow \prod_{\lambda \in \Lambda} \operatorname{Bund}_{*}\left(Y_{\lambda}\right),
$$

which proves property (b). We can now state our main results.

Theorem 12. For any deformation of grouplike quantum groups $\mathscr{H}$ over a connected, simply connected and locally contractible parameter space, there is a pointed 
$\mathrm{CW}$-complex $B \mathscr{H}$ and a universal $\mathscr{H}$-bundle over $B \mathscr{H}$ such that the natural transformation of contravariant functors on $\mathrm{CW}_{*}$,

$$
\begin{aligned}
\eta:[, B \mathscr{H}]_{*} & \rightarrow \operatorname{Bund}_{*}(; \mathscr{H}), \\
\eta_{M}:[M, B \mathscr{H}]_{*} & \rightarrow \operatorname{Bund}_{*}(M ; \mathscr{H}): f \mapsto f^{*} E \mathscr{H}
\end{aligned}
$$

is a natural equivalence.

We would of course like to have an analogous statement for arbitrary bundles over CW-complexes. Removing the basepoint simply means that we allow free homotopies of bundles and that we forget the isomorphism of $H_{*}$ with one of the fibres. The result is the following.

Theorem 13. For any deformation of grouplike quantum groups $\mathscr{H}$ over a connected, simply connected and locally contractible parameter space, there is a CW-complex $B \mathscr{H}$ and a universal $\mathscr{H}$-bundle E $\mathscr{H}$ such that the natural transformation

$$
\begin{aligned}
\eta:[, B \mathscr{H}] & \rightarrow \operatorname{Bund}(; \mathscr{H}), \\
\eta_{M}:[M, B \mathscr{H}] & \rightarrow \operatorname{Bund}(M ; \mathscr{H}): f \mapsto f^{*} E \mathscr{H}
\end{aligned}
$$

is a natural equivalence.

It might still be interesting to know to what extent something like property (b) is true in the case of arbitrary bundles. More precisely, we want to know in how many different ways one can glue bundles together over some basepoint.

In the classical theory, there is no problem to do this, because all the fibres are the same anyway, but in our situation we first have to make the fibres over the basepoints equal before we can glue. But then which map in $\operatorname{End}_{G_{*}}\left(G_{*}\right)$ shall we use to glue the fibres? The following lemma shows that at most the path component of $\mathbb{X}(\mathscr{H})$ matters.

Lemma 14. Let $\mathscr{P}_{i}$ be $\mathscr{H}$-bundles over $Y_{i}, i=1,2$, having the same fibre over the basepoint. Then all the bundles over $Y_{1} \vee Y_{2}$ are homotopic by based homotopies, provided the maps used to glue the fibres are in the same path component of $\mathbb{X}(\mathscr{H})$.

Proof. We use trivializing coverings of $Y_{1}$ and $Y_{2}$ such that the basepoint meets only one chart and has neighborhoods $V_{1}$ and $V_{2}$ not intersecting any of the other open sets. By Lemma 9 we can even assume that the fibre is constant over $V_{i}$. Let $u_{i}: Y_{i} \rightarrow I$ be continuous functions with support in $V_{i}$ and $u_{i}(*)=1$. Furthermore let $\gamma$ be a path in $\mathbb{X}(\mathscr{H})$.

We construct a bundle $\mathscr{P}$ over $\left(Y_{1} \vee Y_{2}\right) \times I$ as follows. Over $Y_{1} \times I$ we take the bundle $\mathscr{P}_{1}$ with the exception that in $(y, t) \in V_{1} \times I$ we take $H_{\mathrm{pr}_{1} \circ \gamma\left(t u_{1}(y)\right)}$ as the structure group. This does not affect the coordinate changes. Over $Y_{2} \times I$ we introduce an additional chart, let $U_{0}$ be the unique chart of $Y_{2}$ that meets the basepoint. We replace it by $U:=U_{0} \backslash\{*\}$ and introduce $V$ as a new chart. For the structure group over $V \times I$ we use $H_{\mathrm{pr}_{1} \circ \gamma\left(t u_{2}(y)\right)}$ over the point $(y, t) \in V \times I$. For the coordinate change over $V \backslash\{*\}$ we use the one associated to the section $(y, t) \mapsto \gamma\left(t u_{2}(y)\right)^{-1}$ of the family of character groups over $(V \backslash\{*\}) \times I$. We now glue the bundles together along $\{*\} \times I$ using the identity. Then $\mathscr{P}$ is a homotopy from the bundle obtained by glueing by means of $\gamma(0)$ to the one obtained by glueing by means of $\gamma(1)$. 
Unfortunately, this homotopy does not preserve the basepoint, because the fibre over the basepoint varies during the homotopy. $\left.\mathscr{P}\right|_{\{*\} \times I}$ is a bundle over a circle. By using the same method as in Lemma 9 we can change the structure group in a small neighborhood of $\{*\} \times I$ by contracting the image of $\{*\} \times I$ to the basepoint, which is possible by the simple connectedness of $X$.

4.5. Homotopy Groups of Classifying Spaces. In the classical case, the classifying space operator (or functor rather) shifts the homotopy groups by $1: \pi_{n}(B G)=$ $\pi_{n-1}(G)$. We want to remark that this remains true in the quantum case if " $\pi_{n}(\mathscr{H})$ " is suitably interpreted.

Consider $\pi_{0}$ first. The group $\pi_{1}(B \mathscr{H})$ classifies pointed $\mathscr{H}$-bundles on $S^{1}$, but they are easy to describe in terms of charts. The following result justifies calling this group $\pi_{0}(\mathscr{H})$.

Proposition 15. The group $\pi_{1}(B \mathscr{H})$ is the quotient of the free group generated by the set $\pi_{0}(\mathbb{X}(\mathscr{H}))$ by the following relation: whenever the intersection of the projections of two components $c_{1}, c_{2} \subset \mathbb{X}(\mathscr{H})$ to the parameter space is nonempty, they can be represented by elements of a character group for one of the common parameters, the product $c_{1} c_{2}$ is then the component of the product of these representatives.

It is now easy to guess what the higher homotopy groups of $B \mathscr{H}$ are:

Proposition 16. The higher homotopy groups of $B \mathscr{H}$ are given by $\pi_{n}(B \mathscr{H})=$ $\pi_{n-1}\left(\mathbb{X}(\mathscr{H})^{0}\right)$, where $\mathbb{X}(\mathscr{H})^{0}$ denotes the path component of the identity in $\mathbb{X}(\mathscr{H})$.

If we write $\pi_{n}(\mathscr{H})$ for $\pi_{n}\left(\mathbb{X}(\mathscr{H})^{0}\right)$, we recover the formula $\pi_{n}(B \mathscr{H}) \cong \pi_{n-1}(\mathscr{H})$, in complete analogy to the classical case.

\section{Twisted $S U(n)$}

In [9] Woronowicz has given an example of a deformation of the group $S U(n)$. The parameter space in this case is $X=] 0,1]$, and for every value $\mu \in X$, he defines a quantum group $S U_{\mu}(n)$. These quantum groups form a continuous deformation, to which we wish to apply the theory of the preceding sections.

5.1. Character Group of $S U_{\mu}(n)$. The quantum group $S U_{\mu}(n)$ of Woronowicz [9] is a $C^{*}$-algebra generated by elements $u_{i k}, 1 \leqq i, k \leqq n$, with the following relations. Let $\sigma \in S_{n}$ be a permutation, then $I(\sigma)$ is the number of inversions:

$$
I(\sigma)=|\{(i, j) \mid i<j \wedge \sigma(i)>\sigma(j)\}| .
$$

There are relations which justify the " $U$ " in " $S U_{\mu}(n)$ "

$$
\sum_{k} u_{m k} u_{l k}^{*}=\delta_{l m} I, \quad \sum_{k} u_{k l}^{*} u_{k m}=\delta_{l m} I
$$

and relations generalizing the determinant condition (the " $S$ " of " $S U_{\mu}(n)$ ")

$$
\begin{aligned}
\sum_{\sigma \in S_{n}}(-\mu)^{I(\sigma)} u_{\sigma(1), \tau(1)} \cdots u_{\sigma(n), \tau(n)}=(-\mu)^{I(\tau)} & \forall \tau \in S_{n}, \\
\sum_{\sigma \in S_{n}}(-\mu)^{I(\sigma)} u_{\sigma(1), k_{1}} \cdots u_{\sigma(n), k_{n}}=0 & \forall\left\{k_{1}, \ldots, k_{n}\right\} \subsetneq\{1, \ldots, n\},
\end{aligned}
$$

where the parameter $\mu$ ranges over $X$. 
Suppose $\xi \in \mathbb{X}\left(S U_{\mu}(n)\right)$, then it is mapped under $\varphi$ to a matrix $x_{i k}=\xi\left(u_{i k}\right)$. The matrix $x$ is unitary because of (2), and in addition the entries have to satisfy the relations

$$
\sum_{\sigma \in S_{n}}(-\mu)^{I(\sigma)} x_{\sigma(1), \tau(1)} \cdots x_{\sigma(n), \tau(n)}=(-\mu)^{I(\tau)} \quad \forall \tau \in S_{n} .
$$

If $\mu=1$, then all the relations are identical, and they only say that the matrix $x$ has determinant equal to 1 . The relations (4) are trivially verified; because at least two of the $k_{i}$ are equal, the formula (4) with $u$ replaced by $x$ just computes the determinant of a matrix with at least two equal columns, which is zero. The character group of $S U_{1}(n)$ is therefore nothing else but $S U(n)$.

Note that the diagonal matrices in $T^{n-1}=\left\{\operatorname{diag}\left(\lambda_{1}, \ldots, \lambda_{n}\right) \mid \lambda_{1} \cdots \lambda_{n}=1\right\}$ are in the character group $\mathbb{X}\left(S U_{\mu}(n)\right)$ for any $\mu$, because for these matrices the relations (5) reduce to

$$
\sum_{\sigma \in S_{n}}(-\mu)^{I(\sigma)} x_{\sigma(1), \tau(1)} \cdots x_{\sigma(n), \tau(n)}=\sum_{\sigma \in S_{n}}(-\mu)^{I(\sigma)} \delta_{\sigma, \tau}=(-\mu)^{I(\tau)} \quad \forall \tau \in S_{n} .
$$

Again the relations (4) are trivially satisfied, because in every term of the sum there appears at least one nondiagonal and hence vanishing factor. We want to show that there are no other matrices in the character group, for most values of the parameter. For this the relations (5) suffice.

Proposition 17. There is an interval in $X$ containing 1 such that $\mathbb{X}\left(S U_{\mu}(n)\right)=T^{n-1}$ except at $\mu=1$ where $\mathbb{X}\left(S U_{1}(n)\right)=S U(n)$.

Proof. The relations (5) form a system of linear equations for the $n$ ! products $x_{1 \sigma(1)} \cdots x_{n \sigma(n)}$, where $\sigma$ ranges over all permutations. The coefficient matrix of this system is $(-\mu)^{I\left(\sigma \tau^{-1}\right)}$. The solutions are functions on $S_{n}$, e.g. the matrices in $T^{n-1}$ are solutions, they correspond to the indicator function of the identity element. The determinant of the coefficient matrix is a polynomial in $\mu$ with constant term \pm 1 and degree $n !\left(\begin{array}{l}n \\ 2\end{array}\right)$. Because its zeros are isolated, there is a connected neighborhood of 1 such that the indicator function of the identity is the only solution:

$$
\begin{aligned}
x_{11} \cdots x_{n n} & =1, \\
x_{1 \sigma(1)} \cdots x_{n \sigma(n)} & =0 \quad \forall \sigma \in S_{n} \backslash\{e\} .
\end{aligned}
$$

(7) says that $x$ cannot be a matrix obtained from one in $T^{n-1}$ by conjugation by some permutation matrix, because then all the products $x_{1 \sigma(1)} \cdots x_{n \sigma(n)}$ would be zero unless $\sigma$ is the permutation corresponding to the permutation matrix, for which the product would be 1 . These are exactly the matrices of the normalizer of $T^{n-1}$ in $S U(n)[1]$.

(6) and (7) also imply that the determinant of $x$ is 1 , because

$$
\operatorname{det}(x)=\sum_{\sigma \in S_{n}} \operatorname{sign}(\sigma) x_{1 \sigma(1)} \cdots x_{n \sigma(n)}=x_{11} \cdots x_{n n}=1 .
$$

Next we show that the only matrices satisfying the relations (6) and (7) are the matrices in $T^{n-1}$. We know that $\mathbb{X}\left(S U_{\mu}(n)\right)$ is a Lie group with maximal torus $T^{n-1}$, the torus cannot be larger than that of $S U(n)$ by the preceding paragraph. Suppose its dimension is larger than $n-1$. Then there is a vector $a^{\prime}$ in $\mathfrak{s u}(n)$ 
complementary to $t$ such that $\exp (t a) \in \mathbb{X}\left(S U_{\mu}(n)\right)$ for all $t$. $a$ is a traceless antihermitian matrix, and we can even assume that all the diagonal entries vanish. Therefore there exists a symmetric pair of nonzero entries off the diagonal, $x_{i j}$ and $x_{j i}$ say. Then the same entries of $\exp (t a)$ are nonzero, at least for sufficiently small values of $t$. Then the product corresponding to the transposition of $i$ and $j$ will be nonzero, contradicting the relations (7). The connected component of the identity of $\mathbb{X}\left(S U_{\mu}(n)\right)$ is therefore $T^{n-1}$, so $\mathbb{X}\left(S U_{\mu}(n)\right)$ must be a subgroup of the normalizer of $T^{n-1}$ in $S U(n)$, but as we saw above, no element of the normalizer outside $T^{n-1}$ is in $\mathbb{X}\left(S U_{\mu}(n)\right)$. This completes the proof.

5.2. Classifying Space of $S U_{\mu}(n)$. The very special structure of the family of character groups of $S U_{\mu}(n)$ even allows to compute the classifying space.

Theorem 18. The classifying space of $S U_{\mu}(n)$ over the neighborhood $V$ of Proposition 17 is $B S U(n)$, the "classical" classifying space of $S U(n)$.

Proof. Suppose $\mathscr{P}$ is a $S U_{\mu}(n)$ bundle on $M$ with structure group $\mathscr{G}$. Let $\varphi_{\alpha}$ be a family of bundle charts. The glueing maps $\varphi_{\beta \alpha}$ are functions from $U_{\alpha} \cap U_{\beta}$ into $\mathbb{X}\left(S U_{\mu}(n)\right)$. This last space is homotopy equivalent to $S U(n)$, we can deform the structure group $\mathscr{G}$ together with all the glueing maps so that the structure group becomes the constant $S U(n)$-structure group. Then we just have an $S U(n)$ bundle. But such bundles are classified by homotopy classes of maps into $B S U(n)$.

If we consider only bundles with a fixed quantum group $H=S U_{\mu}(n), \mu$ fixed, as fibre, then the bundles are of course classified by maps into $B \mathbb{X}(H)$. For $\mu \neq 1$ this is $B T^{n-1}$, the associated vector bundles of any representation of $H$ split into a direct sum of line bundles. Allowing the fibres to vary provides us with a much richer theory. For $M=S^{4}$ and $n=2$ we get, e.g.

$$
\begin{aligned}
\operatorname{Bund}_{*}\left(S^{4} ; H\right) & =\left[S^{4}, B S^{1}\right]=H^{2}\left(S^{4} ; \mathbb{Z}\right)=0, \\
\operatorname{Bund}_{*}\left(S^{4} ; S U_{\mu}(n)\right) & =\left[S^{4}, B S U_{\mu}(n)\right]=\pi_{4}(B S U(2))=\pi_{3}(S U(2))=\mathbb{Z} .
\end{aligned}
$$

Note that pointed and unpointed bundles are the same in this case. One can also conclude that a $S U_{\mu}(n)$-bundle having an associated vectorbundle which does not split into linebundles cannot be a $\left(S U_{\mu}(n)\right)_{\mu \in V}$-bundle if $V$ does not contain the parameter value 1 .

It would be interesting to study similarly the deformations of the other classical Lie groups, as Rosso has constructed them in [6].

\section{Conclusion}

We have seen that quite a bit of the theory of principal bundles can be carried over to quantum groups, which allow the fibres to vary, not to drastically though. We have also seen that the quantum group $S U_{\mu}(n)$ of Woronowicz, although it behaves like $S U(n)$ as far as representation theory is concerned, has a completely different homotopy theoretic behavior, at least for parameter values different from 1 . This raises the question whether there exist quantum groups whose representation theory and bundle theory both coincide with that of the group they are supposed to be a deformation of. 
For differential geometric applications it would be interesting to have a concept analogous to connections on the bundles, for this it will be necessary to deform also the differential structure on the quantum group $[10,6]$.

\section{References}

1. Bröcker, Th., tom Dieck, T.: Representations of Compact Lie Groups. Graduate Texts in Mathematics, Vol. 98. Berlin, Heidelberg, New York: Springer 1985

2. Dieudonné, J.: Treatise on Analysis. Pure and Applied Mathematics, Vol. 10. New York: Academic Press 1976

3. Dixmier, J.: Les $C^{*}$-algèbres et leurs représentations. Paris: Gauthier-Villars 1964

4. Maltsiniotis, G.: Groupoïdes quantiques. C.R. Acad. Sci. Paris 314, 249-252 (1992)

5. Manin, Yu.I.: Quantum groups and noncommutative geometry. Montréal: Les publications CRM 1988

6. Rosso, M.: Algèbres enveloppantes quantifiées, groupes quantiques compacts de matrices et calcul différentiel non commutatif. Duke Math. J. 61, 11-40 (1990)

7. Spanier, E.H.: Algebraic Topology. New York: McGraw-Hill 1966

8. Woronowicz, S.L.: Compact Matrix Pseudogroups. Commun. Math. Phys. 111, 613-665 (1987)

9. Woronowicz, S.L.: Tannaka-Krein Duality for compact matrix pseudogroups. Invent. Math. 93, 35-76 (1988)

10. Woronowicz, S.L.: Differential calculus on compact matrix pseudogroups. Commun. Math. Phys. 122, 125-170 (1989)

Communicated by K. Gawedzki 